\title{
CONNECTIONS BETWEEN THE MATCHING AND CHROMATIC POLYNOMIALS
}

\author{
E.J. FARRELL \\ Department of Mathematics \\ The University of the West Indies \\ St. Augustine, Trinidad \\ and \\ EARL GLEN WHITEHEAD, JR. \\ Department of Mathematics \& Statistics \\ University of Pittsburgh \\ Pittsburgh, PA 15260 U.S.A.
}

(Received June 8, 1990 and in revised form June 6, 1991)

\begin{abstract}
The main results established are (i) a connection between the matching and chromatic polynomials and (ii) a formula for the matching polynomial of a general complement of a subgraph of a graph. Some deductions on matching and chromatic equivalence and uniqueness are made.
\end{abstract}

KEY WORDS AND PHRASES. Matching polynomial, chromatic polynomial, matching equivalence, chromatic equivalence, triangle-free graphs.

1991 AMS SUBJECT CLASSIFICATION CODE. 05A99, 05 C99.

\section{INTRODUCTION.}

The graphs considered here are finite, undirected, and contain no loops and no multiple edges. Let $G$ be such a graph. A matching in $G$ is a spanning subgraph of $G$, whose components are nodes and edges only. Let $a_{k}$ be the number of matchings in $G$ with $k$ edges and let $n$ be the number of nodes in $G$. Then the matching polynomial of $G$ is

$$
M(G ; \underline{w})=\sum_{k=0}^{[n / 2]} a_{k} w_{1}^{n-2 k} w_{2}^{k}
$$

where $w_{1}$ and $w_{2}$ are indeterminates or weights associated with each node and edge respectively, in $G$. If we put $w_{1}=w_{2}=w$, then we obtain

$$
M(G ; w)=\sum_{k=0}^{[n / 2]} a_{k} w^{n-k},
$$

which is called the simple matching polynomial of $G$. The basic properties of the matching polynomial can be found in the introductory paper by Farrell [1].

The chromatic polynomial of a graph $G$ is the polynomial $P(G ; \lambda)$ which represents the number of ways of coloring the nodes of $G$ with $\lambda$ colors, in such a way that adjacent nodes receive different 
colors. The basic properties of $P(G ; \lambda)$ can be found in Read [10]. In this paper, we will assume that $P(G ; \lambda)$ is expressed in the complete graph basis, i.e.,

$$
P(G ; \lambda)=\sum_{k=0}^{n} c_{k}(\lambda)_{n-k}
$$

where $c_{k}$ is the number of color partitions of the nodes of $G$ into $n-k$ non-empty indistinguishable classes and $(\lambda)_{r}=\lambda(\lambda-1)(\lambda-2) \cdots(\lambda-r+1)$.

Let $\underline{m}(G)$ denote the vector of the nonzero coefficients of $M(G ; \underline{w})$, written in ascending powers of $w_{2}$. This vector is called the matching vector of $G$. Graphs $G$ and $H$ are matching equivalent if $\underline{m}(G)=\underline{m}(H)$. Analogously, let $\underline{c}(G)$ denote the vector of the nonzero coefficients of $P(G ; \lambda)$. This vector is called the chromatic vector of $G$. Graphs $G$ and $H$ are chromatically equivalent if $\underline{c}(G)=\underline{c}(H)$. Let $V(G)$ denote the node set of $G$ and $|V(G)|$ denote the cardinality of $V(G)$. Graphs $G$ and $H$ are called co-matching if $|V(G)|=|V(H)|$ and $G$ and $H$ are matching equivalent. Graphs $G$ and $H$ are called co-chromatic if $|V(G)|=|V(H)|$ and $G$ and $H$ are chromatically equivalent. A graph $G$ is matching unique if $M(G ; \underline{w})=M(H ; \underline{w})$ implies that $H$ is isomorphic to $G$. The term chromatically unique is analogously defined.

A chain is a tree with nodes of valencies 1 and 2 only. The chain with $n$ nodes will be denoted by ' $P_{n}$. The notations $K_{p}, K_{m, n}$ and $C_{p}$ will be used for the complete graph with $p$ nodes, the complete $m$ by $n$ bipartite graph and the cycle (circuit) with $p$ nodes, respectively. Let $S$ be a subset of $V(G)$. Then $G-S$ will denote the graph obtained from $G$ by removing the nodes in $S$. If $S$ is a subset of $E(G)$, then $G-S$ will denote the graph obtained from $G$ by removing the edges in $S$.

We extend a result of Frucht and Giudici [3], by showing that their necessary condition for the matching vector to be equal to the chromatic vector is also a sufficient condition. A formula for the matching polynomial of a complement of a subgraph of a labeled graph is then derived. Also, we obtain connections between matching and chromatic equivalence and uniqueness. Finally, we deduce some results for $\theta$-graphs.

In the material which follows, the upper and lower limits of summations will be omitted when they are obvious from the contents of the summand. For example, the lower summation limit is zero and the upper summation limit is $[n / 2]$ in all matching polynomials of graphs with $n$ nodes. The notation $G_{1} \cup G_{2} \cup \cdots \cup G_{r}$ will denote the disjoint union of graphs $G_{1}, G_{2}, \ldots$ and $G_{r}$. The notation $(G, H)$ will be used for a pair of two equivalent, co-matching or co-chromatic graphs; this pair will be referred to as an equivalent, co-matching or co-chromatic pair, as appropriate.

2. CONNECTIONS BETWEEN THE MATCHING AND THE CHROMATIC POLYNOMIALS.

The following lemmas can be easily proved.

LEMMA 1. Every proper coloring of a graph $G$ with $r$ colors induces a partition of $V(G)$ into $r$ parts in which nodes $x$ and $y$ belong to a part only if $x y \notin E(G)$.

LEMMA 2. Let $G$ be a graph (without loops or multiple edges). Then

$$
P(G ; \lambda)=\sum_{k} a_{k}(\lambda)_{p-k},
$$

where $a_{k}$ is the number of partitions of $V(G)$ into exactly $p-k$ parts such that nodes $x$ and $y$ belong to the same part only if $x y \notin E(G)$; and the summation is taken over all (nonnegative integral) values of $k$ less than $p$.

DEFINITION. A color partition of $G$ is a partition of $V(G)$ induced by a proper coloring of $G$. 
LEMMA 3. Let $\left\{R_{1}, R_{2}, \ldots, R_{n}\right\}$ be the set of all color partitions of $V(G)$, where $R_{k}=\left\{S_{1, k}, S_{2, k}, \ldots, S_{r_{k}, k}\right\}$. Then $\left|S_{i, j}\right| \leq 2$ for all integers $i$ and $j$ such that $1 \leq i \leq r_{j}$ and $1 \leq j \leq n$, if and only if $\bar{G}$ is $\Delta$-free (triangle free). Hence, if $P(G ; \lambda)=\sum_{k} a_{k}(\lambda)_{p-k}$. Then $a_{k}$ is the number of matchings in $\bar{G}$ with $p-k$ components if and only if $\bar{G}$ is $\Delta$-free.

PROOF. Suppose that $\bar{G}$ is $\Delta$-free. Let us assume that $\left|S_{i, j}\right|>2$ for some $i$ and $j$. Let $x, y, z \in S_{i, j}$. Then $x, y$ and $z$ are nonadjacent in $G$. Thus, $x y z$ is a triangle in $\bar{G}$. This is a contradiction. Therefore, our assumption is false. $\left|S_{i, j}\right| \leq 2$ for all $i$ and $j$.

Conversely, suppose that $\left|S_{i, j}\right| \leq 2$ for all $i$ and $j$. Then $G$ does not contain a proper coloring in which three nodes can be colored the same. Thus, $G$ does not contain a set of three mutually nonadjacent nodes. Therefore, $\bar{G}$ is $\Delta$-free. This proves the first part of the theorem. When $\left|S_{i, j}\right| \leq 2$ for all $i$ and $j$, the subgraph of $G$ induced by $S_{i, j}$ is either a node (if $\left|S_{i, j}\right|=1$ ) or an edge (if $\left|S_{i, j}\right|=2$ ). It follows that each color partition of $V(G)$ induces a unique matching in $G$.

Since the number of matchings in $\bar{G}$ with $r$ components is the coefficient of $w^{r}$ in the simple matching polynomial of $\bar{G}$, we immediately obtain the following theorem.

THEOREM 1. Let $G$ be a graph. Then $P(G ; \lambda)=M\left(\bar{G} ; w^{\prime}\right)$ (where $w^{\prime}$ means that $w^{k}$ in $M(G ; w)$ is replaced by $\left.(\lambda)_{k}\right)$ and dually, $M(\bar{G} ; \underline{w})=P\left(G ; \lambda^{\prime}\right)$ (where $\lambda^{\prime}$ means that $(\lambda)_{k}$ is replaced by the monomial $w_{1}^{2 k-p} w_{2}^{p-k}$ and $p$ is the number of nodes in $G$ ) if and only if $\bar{G}$ is $\Delta$-free.

COROLLARY 1.1. $\underline{m}(G)=\underline{c} \bar{G})$ if and only if $G$ is $\Delta$-free.

This corollary is an improvement of the main result in [5], since it gives both a necessary and sufficient condition for the matching vector of a graph to be equal to the chromatic vector of the complement graph.

Suppose that $\bar{G}$ is not $\Delta$-free. Then the sets $S_{i, j}$ may have more than two elements. In this case, for some $k$, there will be other $k$-color partitions of $V(G)$ (viz those which contain $S_{i, j}$ 's for which $\left.\left|S_{i, j}\right|>2\right)$. This observation and Theorem 1 yield the following result.

THEOREM 2. Let $G$ be a graph;

$$
M(G ; w)=\sum_{k} b_{k} w^{p-k}
$$

its simple matching polynomial and

$$
P \bar{G}) ; \lambda)=\sum_{k} a_{k}(\lambda)_{p-k}
$$

the chromatic polynomial of its complement. Then $a_{k} \geq b_{k}$, for all values of $k$. Furthermore, equality holds (for all values of $k$ ) if and only if $G$ is $\Delta$-free.

We note that Theorem 2, not only characterizes graphs whose matching vector is equal to the chromatic vector of its complement, but also gives (i) a lower bound for the coefficients of the chromatic polynomial and (ii) a criterion for determining whether or not a given graph contains triangles.

\section{MATCHING POLYNOMIALS OF COMPLEMENTS OF GRAPHS.}

DEFINITION. Let $H$ be a graph and $G$ a subgraph of $H$. A complement of $G$ in $H$ is a graph obtained from $H$ by removing the edges of an isomorph of $G$.

Thus, if $R$ is a complement of $G$ in $H$, then $V(R)=V(H)$ and $E(R)=E(H)-E\left(G^{\prime}\right)$, where $G^{\prime}$ is an isomorph of $G$. Since $H$ is unlabeled, there could be, in general, several complements of $G$ in $H$. However, if we take $H$ to be a labeled graph, then there is only one (labeled) complement of $G$ 
in $H$. In this case, we denote the unique complement of $G$ in $H$ by $G_{H}$. If we take $H$ to be the complete graph $K_{p}$, then again the complement is unique and is denoted by $\bar{G}_{p}$, or simply by $\bar{G}$, when it is unnecessary to specify the number of nodes. Notice that if in addition, $G$ also has $p$ nodes, then the complement is the usual graph complement. If $H$ is the labeled bipartite graph $K_{m, n}$, then we denote the complement by $\bar{G}_{m, n}$, or $\bar{G}_{B}$, where $B$ is a complete bipartite graph, if it is unnecessary to specify the cardinalities of the disjoint node sets.

The following theorem gives the matching polynomial of the complement of a graph $G$ relative to any labeled graph $H$ which contains $G$ as a subgraph.

THEOREM 3. Let $G$ be a graph with $e$ edges. Let $H$ be a labeled graph containing $G$ as a subgraph. Then

$$
M\left(G_{H} ; \underline{w}\right)=\sum_{k=1}^{e}(-1)^{k} w_{2}^{k} \sum M\left(H-V\left(S_{k}\right) ; \underline{w}\right),
$$

where $S_{k}$ is a set of $k$ edges of $G$ belonging to a matching in $H$ and the second summation is taken over all such matchings in $H$ containing $k$ edges of $G$.

PROOF. Our proof is based on the Principle of Inclusion and Exclusion. Let $p_{i}$ be the property that the edge $i$ of $G$ is used in the $a$ matching of $H$. Then there are $e$ properties -- one for each edge in $G$. Let $N\left(p_{1}, p_{2}, \ldots, p_{k}\right)$ denote the contribution to $M(H ; \underline{w})$ of the matchings containing the edges $1,2, \ldots, k$ of $G$. We must find $N\left(p_{1}^{\prime}, p_{2}^{\prime}, \ldots, p_{e}^{\prime}\right)$, i.e., the contribution to $M(H ; \underline{w})$ of the matchings which do not contain any of the $e$ edges of $G$. Therefore, this is the contribution of all the matchings in $\bar{G}_{H}$, i.e., $M\left(\bar{G}_{H} ; \underline{w}\right)$.

Let $D_{k}$ be a matching in $H$ which contains the set $S_{k}=\left\{i_{1}, i_{2}, \ldots, i_{k}\right\}$ of $k$ edges of $G$. The edges in $S_{k}$ are independent and therefore cover $2 k$ nodes of $H$. Suppose that $H$ contains $n$ nodes. Then the remaining $n-2 k$ nodes of $H$ can be covered by a matching $\alpha_{k}$ of $H-V\left(S_{k}\right)$. Clearly, no edge of $\alpha_{k}$ can be incident to any of the $k$ edges in $G$.

Therefore the matchings which cover the $n-2 k$ nodes can be enumerated independently. They will be all the matchings in $H-V\left(S_{k}\right)$. Therefore, the contribution of $D_{k}$ to the matching polynomial of $H$ is

$$
N\left(p_{1}, p_{2}, \ldots, p_{k}\right)=w_{2}^{k} M\left(H-V\left(S_{k}\right) ; \underline{w}\right)
$$

The contribution of all such matchings of $H$ with $k$ edges belonging to $G$ is therefore

$$
\sum w_{2}^{k} M\left(H-V\left(S_{k}\right) ; \underline{w}\right)
$$

where the summation is taken over all such matchings $D_{k}$ of $H$. The theorem follows by the Principle of Inclusion and Exclusion.

Suppose, in the above theorem, $H$ is the complete graph $K_{n}$. Then $H-V\left(S_{k}\right)$ will be the complete graph $K_{n-2 k}$. If $G$ contains $a_{k}$ matchings with $k$ edges, then the second summation will become $a_{k} M\left(K_{n-2 k} ; \underline{w}\right)$. Thus we have the following result (also essentially given in Zaslavsky [4] and Wahid [5].

COROLLARY 3.1. Let $G$ be a graph with $p$ nodes and $n \geq p$, a positive integer. Also let

$$
M(G ; \underline{w})=\sum_{k}^{[p / 2]} a_{k} w_{1}^{p-2 k} w_{2}^{k} .
$$


Then

$$
M\left(\bar{G}_{n} ; \underline{w}\right)=\sum_{k}(-1)^{k} a_{k} w_{2}^{k} M\left(K_{n-2 k} ; \underline{w}\right) .
$$

In the case where $H$ is the labeled complete bipartite graph $K_{m, n}$, we obtain the following result, also given by Gutman [6].

COROLLARY 3.2. Let $M(G ; \underline{w})=\sum_{k} a_{k} w^{p-2 k} w_{2}^{k}$, where $G$ is a bipartite graph. Then

$$
M\left(\bar{G}_{m, n} ; \underline{w}\right)=\sum_{k}(-1)^{k} a_{k} w_{2}^{k} M\left(K_{m-k, n-k} ; \underline{w}\right) .
$$

We note from Corollary 3.2 that the matching polynomial of $G_{m, n}$ depends only on the coefficients of the terms in $M(G ; \underline{w})$. It follows that all the complements of $G$ in $K_{m, n}$ are comatching. We therefore speak of the matching polynomial of a complement of a graph in the complete bipartite graph. The graph is not unique but the matching polynomial is.

Corollary 3.1 can be improved as shown in the following result which is essentially given in Godsi [7].

THEOREM 4. Let $G$ be a graph with $p$ nodes. Then

if and only if

$$
M(G ; \underline{w})=\sum_{k} a_{k} w^{p-2 k} w_{2}^{k}
$$

$$
M(\bar{G} ; \underline{w})=\sum_{k}(-1)^{k} a_{k} w_{2}^{k} M\left(K_{p-2 k} ; \underline{w}\right) .
$$

PROOF. Corollary 3.1 establishes the sufficiency of the condition. Assuming the formula for $M(\bar{G} ; \underline{w})$, it can be deducted (after careful manipulations), that $M(G ; \underline{w})$ is the desired formula.

Corollaries 3.1 and 3.2 can be used to obtain results concerning equivalence, co-matching and matching uniqueness. The following are deductions from these two corollaries.

THEOREM 5. If $(G, H)$ is a matching equivalent pair, then so is $\left(G_{n}, H_{n}\right)$. If in addition, $G$ and $H$ are bipartite, then $\left(G_{B}, H_{B}\right)$ is also a matching equivalent pair.

THEOREM 6. $G$ is matching unique if and only if $\bar{G}$ is.

Theorems 5 and 6 can be used to substantially increase the presently known families of equivalent graphs, co-matching graphs and matching unique graphs.

The following lemma is easy to prove.

LEMMA 4. Let $G$ and $H$ be matching equivalent graphs with $p$ nodes and $m$ nodes respectively. Then $M(G ; \underline{w})=w_{1}^{r} M(H ; \underline{w})$, where $r=p-m$.

THEOREM 7. Let $G$ be a graph which is matching equivalent to a $\Delta$-free graph $H$. Then

$$
P(\bar{H} ; \lambda)=M\left(G ; w^{*}\right)
$$

where $w^{*}$ is the transformation in which $w^{k}$ in $M(G ; w)$ is replaced by $(\lambda)_{r+k}$, where $r=|V(H)|-|V(G)|$. Dually, $M(G ; \underline{w})=P\left(\bar{H} ; \lambda^{\prime}\right)$, where $\lambda^{\prime}$ represents the transformation in which $(\lambda)_{r+k}$ is replaced by $w_{1}^{2 r+2 k-p} w_{2}^{p-r-k}$ and $p$ is the number of nodes in $G$.

PROOF. Since $G$ and $H$ are matching equivalent, we have from Lemma 4,

$$
M(G ; \underline{w})=w_{1}^{r} M(H ; \underline{w}), \text { where } r=|V(G)|-|V(H)| \Rightarrow M(G ; w)=w^{r} M(H ; w)
$$


From Theorem 1, we have $M\left(H ; w^{\prime}\right)=P(H ; \lambda)$. From Equation (1), it is clear that the polynomial $M\left(H ; w^{\prime}\right)$ is essentially the polynomial $M(G ; w)$ with $w^{k}$ replaced by $(\lambda)_{r+k}$. Hence the result follows. The dual part of the proof can be similarly established.

We illustrate the theorem with the following example. Let $G$ and $H$ be the graphs shown in Figure 1. In this example, $r=0$ because both $G$ and $H$ have 5 nodes.

FIGURE 1:

The following polynomials are easily computed.

and

$$
M(G ; \underline{w})=w_{1}^{5}+5 w_{1}^{3} w_{2}+4 w_{1} w_{2}^{2}
$$

$$
P(G ; \lambda)=(\lambda)_{5}+5(\lambda)_{4}+4(\lambda)_{3}=M\left(G ; w^{*}\right)
$$

Notice that $G \cong \bar{H}$. Also, it can be verified that

and

$$
P(\bar{G} ; \lambda)=P(H ; \lambda)=(\lambda)_{5}+5(\lambda)_{4}+5(\lambda)_{3}+(\lambda)_{2}
$$

$$
M(H ; \underline{w})=M(G ; \underline{w})=w_{1}^{5}+5 w_{1}^{3} w_{2}+4 w_{1} w_{2}^{2}
$$

Thus $G$ and $H$ are matching equivalent graphs. Hence $G$ is matching equivalent to a $\Delta$-free graph $H$. However, $M\left(G ; w^{*}\right) \neq P(\bar{G} ; \lambda)$. Thus, if $G$ is matching equivalent to a $\Delta$-free graph, it is the complement of the $\Delta$-free graph (and not the complement of $\underline{G}$ ) that has equal chromatic vector.

It is well known (for example, see [1]) that the characteristic polynomial of a tree coincides with its matching polynomial, except for the alternation of the coefficient signs. This observation, combined with Corollary 1.1, yields the following theorem which gives a relation between the matching, characteristic and chromatic polynomials.

THEOREM 8. For any tree $T, \underline{m}(T)=\underline{\Phi}(T)=\underline{c}(\bar{T})$, where $\underline{\Phi}(T)$ is the vector of the absolute values of the coefficients of the characteristic polynomial of $T$.

Suppose that we take $T$ to be the chain $P_{n}$ with $n$ nodes. From [1] (Theorem 9), we have

$$
M\left(P_{n} ; \underline{w}\right)=\sum_{r}\left(\begin{array}{c}
n-r \\
r
\end{array}\right) w_{1}^{n-2 r} w_{2}^{r}
$$

It is well known (see Farrell [8] Corollary 7.1) that

$$
\Phi\left(P_{n} ; x\right)=\sum_{r}\left(\begin{array}{c}
n-r \\
r
\end{array}\right)(-1)^{r} x^{n-2 r} .
$$

From Theorem 7, we have

$$
P\left(\bar{P}_{n} ; \lambda\right)=\sum_{r}\left(\begin{array}{c}
n-r \\
r
\end{array}\right)(\lambda)_{n-r}
$$

These results agree with Theorem 9 in Loerinc [9]. 
Theorem 7 can be used to obtain combinatorial identities when explicit formulas for $P(H ; \lambda)$ and $M\left(G ; w^{*}\right)$ are known. We give an example of this.

EXAMPLE.

$$
(\lambda)_{m}(\lambda)_{n}=\sum_{k=0} \frac{(m)_{k}(n)_{k}}{k !}(\lambda)_{m+n-k} .
$$

PROOF. Since $\overline{K_{m} \cup K_{n}},=K_{m, n}$, it follows (Theorem 8) that

$$
\begin{aligned}
M\left(K_{m, n} ; w^{*}\right) & =P\left(K_{m, n} ; \lambda\right)=P\left(K_{m} \cup K_{n} ; \lambda\right) \\
& =P\left(K_{m} ; \lambda\right) P\left(K_{n} ; \lambda\right)=(\lambda)_{m}(\lambda)_{n} .
\end{aligned}
$$

The results follows by using the formula for $M\left(K_{m, n} ; \underline{w}\right)$ given in [1] (Theorem 20).

We note that this identity was also derived by Goldman et al. in [10] (Corollary 12).

\section{DEDUCTIONS FOR CHROMATIC EQUIVALENCE AND UNIQUENESS.}

The following theorem shows that for $\triangle$-free graphs, chromatic equivalence of complements is completely determined by matching equivalence.

THEOREM 9. Let $G$ and $H$ be $\Delta$-free graphs. Then $(\bar{G}, \bar{H})$ is a chromatically equivalent pair if and only if $(G, H)$ is a matching equivalent pair.

PROOF. Suppose that $(G, H)$ is matching equivalent. Then $\underline{m}(G)=\underline{m}(H)$. Since $G$ and $H$ are $\Delta$-free, $\underline{c}(\bar{G})=\underline{m}(G)$ and $\underline{c}(\bar{H})=\underline{m}(H)$. This implies that $\underline{c}(\bar{G})=\underline{c}(\bar{H})$, which implies that $(\bar{G}, \bar{H})$ is a chromatically equivalent pair.

Conversely, suppose that $(\bar{G}, \bar{H})$ is a chromatically equivalent pair. Then $\underline{c}(\bar{G})=\underline{c}(\bar{H})$. Since $G$ and $H$ are $\Delta$-free, by Theorem 5 we have $\underline{m}(G)=\underline{m}(H)$, i.e., $(G, H)$ is matching equivalent.

It is clear that if $(G, H)$ is a co-matching pair, then (trivially) it is also a matching equivalent pair. If, in addition, $G$ and $H$ are $\Delta$ - free, then by Theorem $9,(\bar{G}, \bar{H})$ is a chromatically equivalent pair. But $G$ and $H$ must have the same number of nodes because they are co-matching graphs. It follows that $\bar{G}$ and $\bar{H}$ have the same number of nodes. Hence $(\bar{G}, \bar{H})$ is a co-chromatic pair. The converse situation is also true, i.e., if $(\bar{G}, \bar{H})$ is a co-chromatic pair, then $(G, H)$ must be a co-matching pair. Hence, we have proven the following theorem.

THEOREM 10. Let $G$ and $H$ be $\triangle$-free graphs. Then $(\bar{G}, \bar{H})$ is, a co-chromatic pair if and only if $(G, H)$ is a co-matching pair.

In Farrell and Wahid [4], many families of co-matching graphs have been identified, and constructions are given for general families. Also, by suitably choosing the lengths of the chains and cycles, these graphs can be made to be $\Delta$-free. Therefore, by applying Theorem 10, many families of co-chromatic graphs can be identified. For example, it can be easily verified that the graphs $G$ and $H$ shown in Figure 2 are co-matching for all $a, b>2$; this was proven in [9]. Note that $G$ and $H$ are $\triangle$-free. Therefore, by Theorem $10,(\bar{G}, \bar{H})$ is a co-chromatic pair.

FIGURE 2: 
An interesting question at this point is the following: Is it possible for two $\Delta$-free graphs to have different chromatic polynomials, but have chromatically equivalent complements? Suppose that there exist $\triangle$-free graphs $G$ and $H$ such that $(G, H)$ is matching equivalent but not chromatically equivalent. Since $G$ and $H$ are $\triangle$-free, $(\bar{G}, \bar{H})$ will be chromatically equivalent by Theorem 9. Our question would be answered if we can find a pair of matching equivalent but chromatically nonequivalent $\triangle$-free graphs. The graphs $G \cong P_{2 n+1}$ and $H \cong P_{n} \cup C_{n+1}$ form such a pair, for all $n>2$. Thus, the answer to our question is yes. We note that $G$ and $H$ were shown to be co-matching in [4]. Their complements were shown to be chromatically equivalent in [9].

Suppose that $G$ is $\triangle$-free and matching unique. Does it follow that $\bar{G}$ will be chromatically unique? The answer is no in general. Let $G$ be the chain $P_{4}$. It has been shown (Farrell and Guo [3]) that $P_{n}$, for $n$ even, is matching unique. Therefore $P_{4}$ is matching unique. However, $P_{4} \cong \bar{P}_{4}$. But $P_{4}$ is not chromatically unique because it is chromatically equivalent to $K_{1,3}$.

Notice that we cannot say that if $G$ is matching unique then $\bar{G}$ is not chromatically unique. For suppose that $G$ is a graph consisting of isolated nodes and independent edges. Then $G$ is known to be matching unique. However, in [9], it was shown that $G$ is chromatically unique.

At this point, let us consider the converse of the above question. Suppose that $\bar{G}$ is chromatically unique, does it follow that $G$ is matching unique? The following theorem answers this question.

THEOREM 11. If $G$ is $\triangle$-free and $G$ is chromatically unique, then $\bar{G}$ is matching unique up to $\Delta$-freeness. That is, there does not exist another $\Delta$-free graph which is matching equivalent to $G$.

PROOF. Let $G$ satisfy the conditions given in the theorem. Suppose that there exists a $\Delta$ free $G_{1}$ such that $M\left(G_{1} ; \underline{w}\right)=M(G ; \underline{w})$. Since $G$ is $\Delta$-free, $M\left(G ; w^{\prime}\right)=P(\bar{G} ; \lambda)$. Since $G_{1}$ is $\Delta$ free, $M\left(G_{1} ; w^{\prime}\right)=P\left(\bar{G}_{1} ; \lambda\right)$. Therefore, $P\left(\bar{G}_{1} ; \lambda\right)=P(\bar{G} ; \lambda)$. Since $G$ is chromatically unique, we must have $\bar{G}_{1} \cong \bar{G}$ which implies that $G_{1} \cong G$.

5. SOME DEDUCTIONS FOR THE THETA GRAPH.

We can use Theorems 1 and 7 in order to derive formulas for the chromatic polynomials of complements of some graphs. For example, if $G$ consists of $m$ isolated nodes and $n$ independent edges, then

$$
P(\bar{G}, \lambda)=\sum_{r}\left(\begin{array}{l}
n \\
r
\end{array}\right)(\lambda)_{m+n+r}
$$

This follows from Theorem 1 and the formula for the matching polynomial of $G$.

We can also obtain the chromatic polynomial of the complement of the graph consisting of two components $P_{n}$ and $C_{n+1}(n>2)$. In this case,

$$
P\left(\overline{P_{n} \cup C_{n+1}} ; \lambda\right)=\sum_{r=0}(2 n+1-r)(\lambda)_{2 n+1-r}=P\left(\overline{P_{2 n+1}} ; \lambda\right) .
$$

In [1], formulas are given for the matching polynomials of the basic graphs with cyclomatic number 2. These graphs are $\Delta$-free for suitable choices of the lengths of the chains and cycles of which they are composed. From Theorem 1, it follows that the chromatic polynomials of the complements of these graphs can be found. For example, ([1] Theorem 14), we have the following matching polynomial for the $\theta$-graph $G(r, s, t)$ consisting of three chains of lengths $r, s$ and $t$ whose endpoints have been identified. Without loss of generality, we assume that $r \leq s \leq t$. 


$$
\begin{aligned}
M(G(r, s, t) ; \underline{w}) & =M\left(P_{r+s+t-1} ; \underline{w}\right)+w_{2}\left[M\left(P_{r-1} ; \underline{w}\right) M\left(P_{s+t-2} ; \underline{w}\right)\right. \\
& +M\left(P_{s-1} ; \underline{w}\right) M\left(P_{r+t-2} ; \underline{w}\right)+M\left(P_{t-1} ; \underline{w}\right) M\left(P_{r+s-2} ; \underline{w}\right) \\
& \left.-M\left(P_{r-1} ; \underline{w}\right) M\left(P_{s-1} ; \underline{w}\right) M\left(P_{t-1} ; \underline{w}\right)\right], \text { where } r, s, t>2 .
\end{aligned}
$$

Clearly, $G(r, s, t)$ is $\Delta$-free, for $r, s, t>2 . \quad$ By Theorem 1 , thus we have

\section{THEOREM 12.}

$$
\begin{aligned}
& P(\bar{G}(r, s, t) ; \lambda)=\sum_{i}\left(\begin{array}{c}
r+s+t-1-i \\
i
\end{array}\right)(\lambda)_{i}+\sum_{i} \sum_{j}\left(\begin{array}{c}
r-1-i \\
i
\end{array}\right)\left(\begin{array}{c}
s+t-2-i \\
j
\end{array}\right)(\lambda)_{i+j+1} \\
& +\sum_{i} \sum_{j}(s-1-i)(r+t-2-i)(\lambda)_{i+j+1}+\sum_{i} \sum_{j}(t-\underset{i}{-i})(r+s-2-i)(\lambda)_{i+j+1} \\
& -\sum_{i} \sum_{j} \sum_{k}\left(\begin{array}{c}
r-1-i \\
i
\end{array}\right)\left(\begin{array}{c}
s-1-j \\
j
\end{array}\right)\left(t-\frac{1}{k}-k\right)(\lambda)_{i+j+k+1}
\end{aligned}
$$

The following theorem shows that the matching polynomial of the $\theta$-graph $G(2, s, t)$ depends only on the sum $s+t$.

THEOREM 13.

$$
M(G(2, s, t) ; \underline{w})=w_{1} M\left(P_{s+t} ; \underline{w}\right)+2 w_{2} M\left(P_{s+t-1} ; \underline{w}\right)+w_{1} w_{2} M\left(P_{s+t-2} ; \underline{w}\right)
$$

PROOF. Applying the fundamental edge theorem (Theorem 1 in [1]) successively to the two edges of the path of length 2 , we obtain

$$
M(G(2, s, t) ; \underline{w})=w_{1} M\left(C_{s+t} ; \underline{w}\right)+2 w_{2} M\left(P_{s+t-1} ; \underline{w}\right)
$$

Applying the fundamental edge theorem to one of the edges of the cycle $C_{s+t}$, we have

$$
M\left(C_{s+t} ; \underline{w}\right)=M\left(P_{s+t} ; \underline{w}\right)+w_{2} M\left(P_{s+t-2} ; \underline{w}\right)
$$

The proof is completed by combining these two equations.

COROLLARY 13.1. The graphs $G\left(2, s_{1}, t_{1}\right)$ and $G\left(2, s_{1}, t_{2}\right)$ are co-matching if and only if

$$
s_{1}+t_{1}=s_{2}+t_{2}
$$

PROOF. If $s_{1}+t_{1}=s_{2}+t_{2}$, then $G\left(2, s_{2}, t_{2}\right)$ are co-matching by Theorem 14 . If $s_{1}+t_{1} \neq s_{2}+t_{2}$, then $\left|V\left(G\left(2, s_{1}, t_{1}\right)\right)\right| \neq\left|v\left(G\left(2, s_{2}, t_{2}\right)\right)\right|$ which implies that $G\left(2, s_{1}, t_{1}\right)$ and $G\left(2, s_{2}, t_{2}\right)$ are not co-matching.

The following corollary appears as Theorem 3.2.4 in [9].

COROLLARY 13.2. The graphs $\overline{\left(G\left(2, s_{1}, t_{1}\right)\right.}$ and $\overline{G\left(2, s_{2}, t_{2}\right)}$ are co-chromatic if and only if $s_{1}+t_{1}=s_{2}+t_{2}$.

PROOF. Since $2 \leq s_{i} \leq t_{i}$ for $i \in\{1,2\}, G\left(2, s_{i}, t_{i}\right)$ is $\Delta$-free. If $s_{1}+t_{1}=s_{2}+t_{2}$, then $\overline{G\left(2, s_{1}, t_{1}\right)}$ and $\overline{G\left(2, s_{2}, t_{2}\right)}$ are co-chromatic by Corollary 13.1 and Theorem 9 . If $s_{1}+t_{1} \neq s_{2}+t_{2}$, 
then $\left|V\left(\overline{G\left(2, s_{1}, t_{1}\right)}\right)\right| \neq\left|V\left(\overline{G\left(2, s_{2}, t_{2}\right)}\right)\right|$ which implies that $\overline{G\left(2, s_{1}, t_{1}\right)}$ and $\overline{G\left(2, s_{2}, t_{2}\right)}$ are not cochromatic.

ACKNOWLEDGEMENT. The first author, E.J. Farrell, would like to thank the CIES and the University of Pittsburgh for their support during the period of research.

\section{REFERENCES}

1. FARRELL, E. J., An Introduction to Matching Polynomials, J. Combin. Theory B, 27 (1979) 75-86.

2. FARRELL, E. J., On a Class of Polynomials Obtained from the Circuits in a Graph and its Application to Characteristic Polynomials of Graphs, Discrete Math., 28 (1979) 121-133.

3. FARRELL, E. J. and GUO, J. M., On the Characterizing Properties of the Matching Polynomial, submitted.

4. FARRELL, E. J. and WAHID, S. A., Some General Classes of Comatching Graphs, Internat. J. Math. and Math., Vol. 11 No. 1 (1988) 87-94.

5. FRUCHT, R. W. and GIUDICI, R. E., A Note on the Matching Numbers of Triangle-Free Graphs, J. Graph Theory, 9 (1985) 455-458.

6. GOLDMAN, J. R., JOICHI, J. T. and WHITE, D. E., Rook Theory III: Rook Polynomials and Chromatic Structure of Graphs, J. Combin. Theory B, 25 (1978) 135-142.

7. GODSIL, C. D., Hermite Polynomials and a Duality Relation for Matching Polynomials, Combinatorica, 1 (1981) 257-262.

8. GUTMAN, I., Ph.D. thesis, University of Belgrade, 1980.

9. LEORINC, B. M., Computing Polynomials for Special Families of Graphs, Courant Institute of Mathematical Sciences, Computer Science Department, New York University, Report NSO-19 (1980).

10. READ, R. C., An Introduction to Chromatic Polynomials, J. Combin. Theory, 4 (1968) 52-71.

11. WAHID, S. A., On Matching Polynomials of Graphs, (M.Phil. Thesis) The University of the West Indies, St. Augustine, Trinidad, (1983).

12. ZASLAVSKY, T., Complementary Matching Vectors and the Uniform Matching Extension Property, European J. Comb., 2(1981) 91-103. 


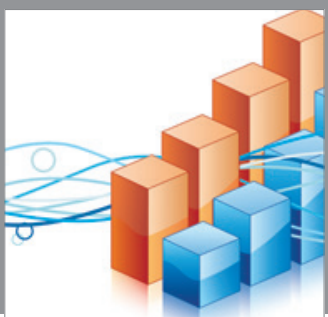

Advances in

Operations Research

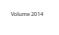

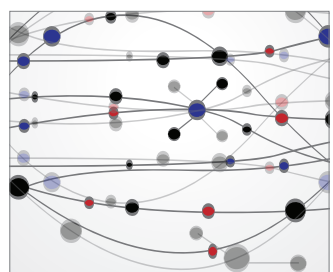

\section{The Scientific} World Journal
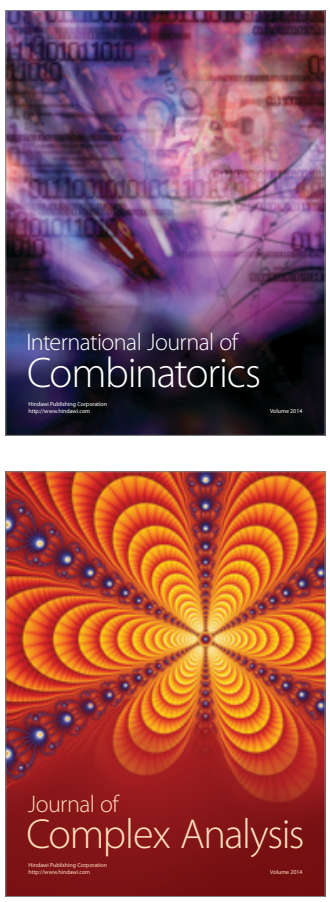

International Journal of

Mathematics and

Mathematical

Sciences


Journal of

Applied Mathematics
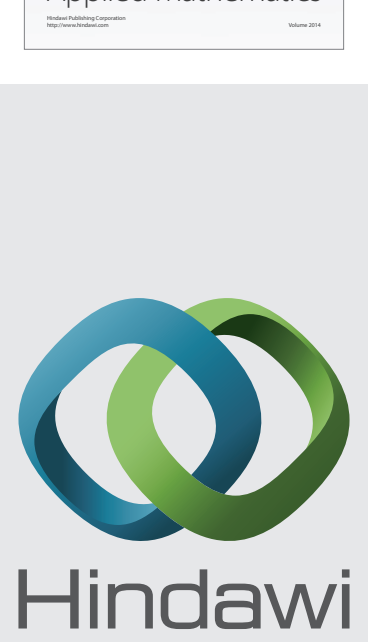

Submit your manuscripts at http://www.hindawi.com
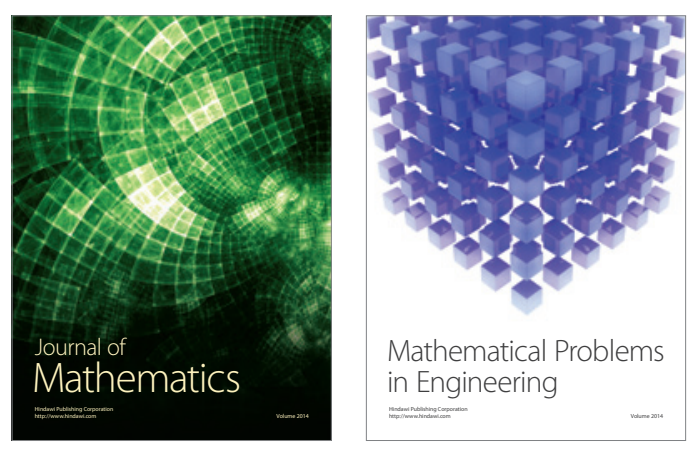

Mathematical Problems in Engineering
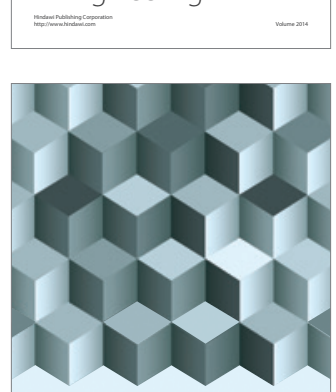

Journal of

Function Spaces
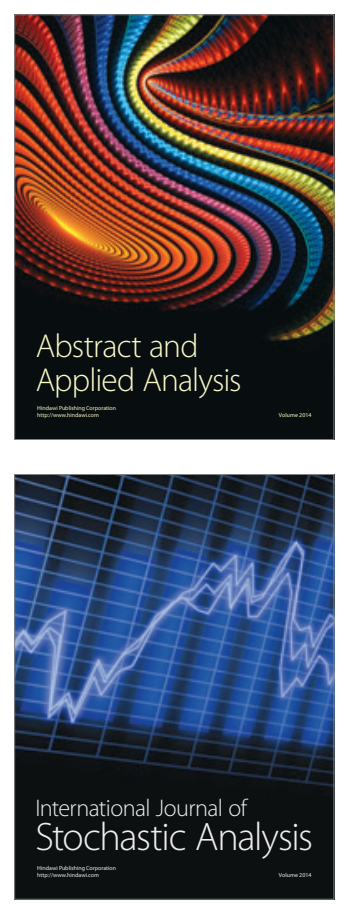

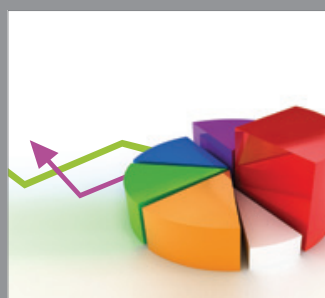

ournal of

Probability and Statistics

Promensencen
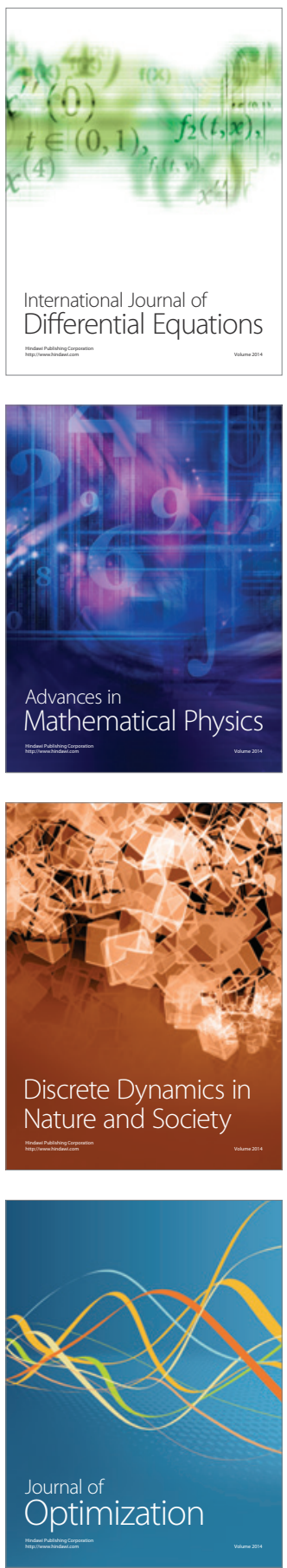